\title{
Chronologie der Online-Enzyklopädie Wikipedia
}

zusammengestellt von Thomas Wozniak

\section{Die Anfänge des Internets}

1957 Als Reaktion auf den Sputnik-Schock geben die USA die Entwicklung des Arpanets (Advanced Research Projects Agency Network) in Auftrag, das ab 1969 verwirklicht wird, als vier Großrechner in vier US-amerikanischen Universitäten miteinander verbunden wurden. 1978 offiziell beendet, wurde der militärische Teil des Arpanets 1990 abgeschaltet.

1990 Öffnung des Internets für kommerzielle Anbieter.

1993 Über das Internet werden ein Prozent der Informationsflüsse der weltweiten Telekommunikationsnetze abgewickelt, erst 2000 überschreitet dieser Wert die Marke von 51 Prozent. (2007 liegt der Wert bei 97 Prozent aller weltweit ausgetauschten Bytes.)

1993 Das Unternehmen Microsoft gibt die Microsoft Encarta, eine elektronische, multimediale Enzyklopädie, heraus, die zunächst auf CD-ROM und DVD, später mit beschränktem Zugriff im Internet verfügbar ist und 2009 eingestellt wird.

1993 Die Idee eines Projekts zur kooperativen Erstellung und Verbreitung einer Enzyklopädie über das Internet mit dem Namen „Interpedia“ wird diskutiert, aber nie umgesetzt.

1994 CD-ROM-Ausgabe der Encyclopædia Britannica, die Online-Version war von Oktober 1999 bis 2001 kostenlos einsehbar, bis die Verluste untragbar wurden; seitdem sind nur noch wenige Sätze einzusehen.

1995 März: Der Programmierer und Pionier des Extreme Programming Howard „Ward“ Cunningham ( ${ }^{\star}$ 1949) gründete das erste Wiki, das Portland Pattern Repository.

2000 März: Jimmy Wales ( ${ }^{\star}$ 1966) und Larry Sanger ( ${ }^{\star}$ 1968) starten über die Firma Bomis die Nupedia, ein englischsprachiges Projekt $\mathrm{zu}$ einer Internet-Enzyklopädie. ${ }^{1}$ Wales hatte Wirtschaft an der University of Alabama und In-

1 Ein Teil der Daten ist extrahiert aus folgenden Artikeln der englischsprachigen Wikipedia: „Censorship of Wikipedia“, „Internet Watch Foundation and Wikipedia“, „List of academic databases and search engines“, „Wikipedia:Milestones“, „Wikipedia timeline“ sowie aus folgenden 
diana studiert und arbeitete als Börsenhändler, bis er sich mit dem Internetdienst Bomis selbstständig machte. Sanger erhielt im Jahr 1991 einen B.A. in Philosophie vom Reed College und 2000 den Ph.D. von der Ohio State University.

\section{Die Anfänge der Wikipedia (2001-2004)}

\section{1}

15. Januar

Das Wiki-System der Nupedia ist unter der eigenständigen Webadresse wikipedia.com erreichbar.

15. März

Jimmy Wales kündigt an, weitere Sprachversionen einrichten zu lassen, unter den ersten sind die deutschsprachige, die katalanische und die japanische Version.

1. April Die Indexierung eines großen Teils der Wikipedia durch Google lenkt einen wachsenden Strom von Lesern zu dem Projekt.

12. Mai Der erste (erhaltene) Artikel der deutschsprachigen Wikipedia „Polymerase-Kettenreaktion“ wird von Magnus Manske erstellt. ${ }^{2}$

August Der erste Medienbericht über Wikipedia erscheint in der Zeitschrift „Wales on Sunday“.

23. Oktober Anlage der Artikel „Erster Weltkrieg“ und „Zweiter Weltkrieg“ durch Kurt Jansson.

24. November Anlage der Empfehlung „Neutraler Standpunkt“ durch Kurt Jansson aufgrund der englischsprachigen Fassung vom 10. November 2001.

2002

Jahresverlauf Das 1947 begründete Nachschlagewerk der Chemie „RÖMPP“ mit 62.000 Stichwörtern und annähernd 200.000 Querverweisen sowie über 12.000 Strukturformeln wird online zugänglich.

26. Februar Abspaltung der Enciclopedia Libre Universal en Español aus Angst vor einer möglichen Kommerzialisierung der damals noch jungen

Artikeln der deutschsprachigen Wikipedia: „Wikipedia:Enzyklopädie/Geschichte der Wikipedia“, „Wikipedia:Meilensteine“ und einigen Meldungen im Archiv des „Wikipedia:Kurier“.

2 Vgl. https://de.wikipedia.org/w/index.php?title=Polymerase-Kettenreaktion\&oldid=2613 (20. 12. 2014). Es handelt sich hierbei um die älteste noch erhaltene Version eines Artikels. Durch eine falsch eingestellte Option der damals verwendeten Wikisoftware wurde die Anzahl der Versionen pro Artikel begrenzt. Nach Erreichen dieser Grenze wurde mit jeder Bearbeitung die jeweils älteste Version des Artikels gelöscht. Welcher Artikel in der deutschsprachigen Wikipedia wirklich der erste war, ist heute nicht mehr feststellbar. 
spanischsprachigen Wikipedia, diese wird seither auf Servern der Universität Sevilla betrieben.

1. März Nach der Kündigung durch Bomis gibt Larry Sanger seine Mitarbeit bei Nupedia auf.

4. Juli Anlage der Empfehlung „Was Wikipedia nicht ist“ durch Helmut Leitner aufgrund der englischsprachigen Fassung vom 24. September 2001.

10. Juli Anlage des Artikels „Geschichte“ durch Benutzer „Ben-Zin“.

27. August Nach Einführung der Qualitätsbewertungsstufe „exzellent“ finden erste Abstimmungsdiskussionen statt.

2003

24. Januar Mit „Löwenzahn“ von Benutzer „Michael w“ überschreitet die deutschsprachige Wikipedia die Marke von 10.000 enzyklopädischen Artikeln.

3. März Anlage der Empfehlung „Wikipedia: Literatur“ führt erstmals zur Einforderung von einheitlich formatierten Literaturhinweisen am Artikelende.

26. Mai Anlage der Empfehlung „Wikipedia: Weblinks“ durch die eine Vereinheitlichung der Formatierungen von Weblinks erreicht werden soll.

20. Juni Gründung der gemeinnützigen „Wikimedia Foundation“ (WMF) durch Jimmy Wales, dieser werden zunächst die Namensrechte, später die Server übereignet.

September Das Projekt „Nupedia“ wird kurz nach dem zweiten Jahrestag eingestellt.

Herbst Kollaborative Schöpfung des Begriffs „Theoriefindung“ statt Original research.

22. November Anlage des „Portal: Geschichte“ durch Benutzer „Sansculotte“.

24. November Einrichtung der Wikisource als freies Schwester-Projekt der Wikipedia zur Sammlung und Transkription von Texten.

Dezember Einer der ersten Edit-Wars in der deutschsprachigen Wikipedia findet bis 14. Januar 2004 im Wikipedia-Artikel „Screening“ statt.

31. Dezember Anlage einer ersten Erklärung zum Begriff „Edit-War“ aufgrund der (nicht mehr vorhandenen) englischsprachigen Version. 


\section{Phase der stabilisierenden Qualitätssicherung (2004-2008)}

\section{4}

30. Januar

12. Februar

24. Februar

April

27. April

2. Juni

13. Juni

7. September
Als weltweit erstes Gericht zitiert das Verwaltungsgericht Göttingen (Aktenzeichen: 2 A 2145/02) die Wikipedia: „Das Arabische gehört zur hamitosemitischen Sprachfamilie (s. die nachfolgende Grafik, zitiert nach Wikipedia der freien Enzyklopädie, www.wikipedia.de).“

Die Server von Wikipedia werden von San Diego in Kalifornien nach Tampa in Florida verlegt.

Der Text „Wikipedia: ,Ich weiß etwas, was du nicht weißt...'“ von Katja Rieger im Magazin „Der Spiegel“3 führt zu einer breiten öffentlichen Rezeption der Internet-Enzyklopädie.

Die „Kamelopedia“, ein auf der MediaWiki-Software basierendes Satire-Wiki wird von Benutzer „Fire“ eingerichtet. Am 10. Dezember 2004 folgt die von Sherrod DeGrippo gegründete „Encyclopedia Dramatica“, am 17. Dezember die vom österreichischen Autor David Sowka gegründete „Stupidedia“ und Anfang 2005 die „Uncyclopedia“ von „Jonathan Huang“.

Anlage der Empfehlungen „Wikipedia: Relevanzkriterien““4 die seither stetig präzisiert werden.

Zum ersten Mal wird die chinesischsprachige Wikipedia für zwei Wochen in der Volksrepublik China blockiert (erneut von Oktober 2005 bis Mitte Oktober 2006). Als Grund wird der 15. Jahrestag der Proteste auf dem Tiananmenplatz im Jahr 1989 angenommen.

Gründung des Vereins „Wikimedia Deutschland - Gesellschaft zur Förderung Freien Wissens“ in Berlin. Mit „Kiebitz (Art)“ von Benutzer „Nina“ und „Opel Laubfrosch“ von Benutzer „Softeis“ überschreitet die deutschsprachige Wikipedia am gleichen Tag die Marke von 100.000 enzyklopädischen Artikeln.

Wikimedia Commons wird eingerichtet, um die Mediendateien sprachversionenübergreifend $\mathrm{zu}$ speichern.

3 Vgl. http://www.spiegel.de/netzwelt/web/wikipedia-ich-weiss-etwas-was-du-nicht-weisst-a-28 7730.html (14.3.2015).

4 Vgl. https://de.wikipedia.org/w/index.php?title=Wikipedia:Relevanzkriterien\&oldid=1169003 (14.3.2015). 
8. September Anlage der Empfehlungen „Wikipedia: Literaturformatierung“, durch die eine Vereinheitlichung der Formatierungen der Literaturtitel erreicht werden soll.

2005

Februar

Das „Historische Lexikon Bayerns“ ein wissenschaftliches OnlineLexikon zur bayerischen Geschichte unter Trägerschaft der Bayerischen Staatsbibliothek München geht online.

22. März Einführung der zweiten Bewertungsstufe „Lesenswert“ unterhalb der „Exzellenten“ Artikel.

April Gründung des Alternativprojekts „Wikiweise“ durch Uli Fuchs, einem ehemaligen Administrator der Wikipedia.

8. April Anlage der Empfehlung „Wikipedia: Allgemeinverständlichkeit“, des damals sogenannten „Oma-Testes“, der eine Verbesserung der Verständlichkeit von Artikeln anstrebt.

30. Juni Wikipedia erhält den „Grimme Online Award“ in der Kategorie Wissen und Bildung.

9. August Mit der Anlage der Empfehlung „Wikipedia: Belege“ durch Klaus Graf soll ein Übergang zur ausschließlichen Einfügung von belegbaren und belegten Informationen erreicht werden. Bis dahin konnten Ergänzungen weitgehend belegfrei eingefügt werden.

22. August Einrichtung des Projektes „Vereins-Wiki“, um Artikeln zu Vereinen, Institutionen und Sportlern eine Plattform zu bieten, die den Relevanzkriterien der Wikipedia (noch) nicht entsprechen.

November Aufgrund von Urheberrechtsverletzungen aufgrund älterer DDRLiteratur, die zwischen November 2003 und Mitte November 2005 in größerem Umfang eingefügt worden war, müssen mehrere hundert Artikel geändert oder gelöscht werden.

November Der Eintrag eines Scherzbolds in Wikipedia, wonach der Journalist John Seigenthaler in die Ermordung des US-Präsidenten Kennedy im Jahr 1963 involviert gewesen sei, löst die Seigenthaler-Kontroverse wurde aus.

15. Dezember Vergleich von je 42 Artikeln verschiedener Disziplinen der englischsprachigen Wikipedia und der Encyclopædia Britannica durch das Wissenschaftsmagazin Nature. Im Schnitt enthielt ein Britannica-Artikel drei Fehler, ein Wikipedia-Artikel vier. ${ }^{5}$

5 Vgl. Giles, Jim: Internet encyclopedias go head to head. In: Nature 438 (2005), S. 900 f.; Giles, Jim: Wikipedia rival calls in the experts, in: Nature 443 (2006), S. 493. Beide Enzyklopädien enthielten acht schwerwiegende Fehler wie falsche Auslegungen wichtiger Theorien. Kleinere 
2006

5. Februar Gründung der „Scholarpedia“ als akademischer Alternative zu Wikipedia durch Eugene Izhikevich, Wissenschaftler am Neurosciences Institute in San Diego. Ebenfalls auf der MediaWikiSoftware basierend, unterliegt sie den Prinzipien von Peer-Review und mit Klarnamen angemeldeten Hauptautoren. ${ }^{6}$

1. März Die englischsprachige Wikipedia überschreitet mit „Jordanhill railway station“ die Marke von 1 Mio. enzyklopädischen Artikeln.

25. März Einrichtung der „Wikipedia: Redaktion Geschichte“ durch Frank Schulenburg, aufgrund des am 26. Januar 2006 gegründeten WikiProjektes „Redaktion Geschichte“.

20. April Aufgrund wiederholter Sperrungen der Wikipedia in der Volksrepublik China wird als Alternative vom chinesischen Suchmaschinenbetreiber Baidu die Internet-Enzyklopädie „Baidu Baike“ geschaffen. $^{7}$

27. April Durch die Anlage der „Vorlage: Internetquelle“ im Namensraum „Vorlage“ soll die Zitierweise von Webseiten vereinheitlicht werden. Die Löschung der Vorlage wurde mehrfach diskutiert und abgelehnt.

11. Mai Als dritte Bewertungsstufe werden die ,informativen Listen und Portale“ eingeführt.

16./17. Juni Die erste Wikipedia-Academy findet in Kooperation mit der Niedersächsischen Staats- und Universitätsbibliothek in Göttingen statt und zielt darauf ab, die Wikipedia stärker im akademischen Umfeld zu etablieren.

16. Juli Der „ArchivBot“, dienstältester und zweitaktivster Bot (automatisiertes Skript) der deutschsprachigen Wikipedia, wird aktiviert.

8. Oktober Durch die Anlage der „Vorlage: Literatur“ im Namensraum „Vorlage“ soll die Zitierweise von Literatur vereinheitlicht werden.

sachliche Fehler, Auslassungen und missverständliche Formulierungen waren in der Britannica 123 und in der Wikipedia 162 enthalten.

6 Jeder Artikel wird von einem Experten auf dem entsprechenden Gebiet geschrieben, der von der Gemeinschaft gewählt oder von einem Redakteur eingeladen wird; jeder Artikel wird einem anonymen Peer-Review unterzogen; jeder Artikel besitzt einen Verantwortlichen, normalerweise den Autor, der Änderung an dem Artikel autorisieren muss.

7 Die Artikel werden von registrierten Nutzern geschrieben und bearbeitet. Jede Bearbeitung wird von Administratoren kontrolliert. Für die Nutzer besteht keine Möglichkeit, die Administratoren zu kontaktieren. Die Beiträge werden in einem Punktesystem belohnt. 
Die Löschung der Vorlage wurde mehrfach diskutiert und abgelehnt.

26. Oktober In Schweden startet das rechtsextreme Online-Lexikon „Metapedia“ auf Wikibasis mit mittlerweile 16 Sprachversionen (2014: 50.000 deutschsprachige Artikel). Den 280.000 ungarischen Wikipedia-Artikeln stehen 140.000 ungarische Metapedia-Artikel gegenüber.

11. November Mit der „Zimmerischen Chronik“ wurde das erste Großprojekt der deutschsprachigen Wikisource vollendet. Seither wird die wissenschaftliche Aufbereitung der deutschsprachigen Wikisource durch korrekte Textangaben und kollaboratives Korrekturlesen der gescannten oder transkribierten Texte verstärkt.

23. November Mit „Janina Korowicka“ von Marcus Cyron überschreitet die deutschsprachige Wikipedia die Marke von 500.000 enzyklopädischen Artikeln.

23. November In Tunesien wird die Webseite von Wikimedia bis 27. November blockiert.

Dezember Ein deutscher Comedian, der als Kunstfigur „Atze Schröder“ auftritt, verklagt Arne Klempert, den (damaligen) Vorsitzenden von Wikimedia Deutschland e.V., weil er seinen echten Namen nicht in Wikipedia veröffentlichen will. „Schröder“ zieht seine Klage zwar zurück, will die Rechtsanwaltskosten aber von Klempert bezahlt bekommen. Ein Gericht weist den Anspruch ab.

Jahresverlauf Online-Veröffentlichung von etwa 150.000 Artikeln aus Meyers Lexikon in 24 Bänden unter dem Namen „Meyers Lexikon online“. Trotz der nach Verlagsangaben 14 Mio. Aufrufe im Monat und einer umfangreichen Überarbeitung zum 23. September 2008 wird das kostenlose Angebot am 23. März 2009 eingestellt.

2007

Frühjahr Der bisherige Höhepunkt der Zahl der Bearbeitungen sowie der Anmeldungen wird erreicht und sinkt seither kontinuierlich.

14. Januar Einrichtung des von Wikimedia Deutschland e.V. finanzierten „Literaturstipendiums“ durch Denis Barthel: Nach dem Konzept einer dezentralen Bibliothek werden literarische Werke finanziert, bei Benutzern gelagert und für die weitere Verarbeitung in Artikeln organisiert.

23. Januar Die DBpedia, ein Gemeinschaftsprojekt der Universität Leipzig, der Universität Mannheim, des Hasso-Plattner-Instituts und „OpenLink Software“, die strukturierte Informationen aus Wi- 
Februar Die vorgetäuschte Identität des Benutzers „Essjay“, eines Benutzerkonto-Verwalters der englischsprachigen Wikipedia, fliegt auf. „Essjay“ war Administrator, Mitglied des Arbitration Committee und Community Manager. Der vermeintlich promovierte Theologe, Doktor des kanonischen Rechts und Professor einer Privatuniversität im Osten der USA entpuppte sich als 24-jähriger Student und Rechtsanwaltsgehilfe. Jimmy Wales kündigte daraufhin eine Klarnamen-Initiative an.

16. Februar Der türkische Historiker Taner Akçam wird auf dem Internationalen Flughafen Montréal-Pierre Elliott Trudeau vorübergehend festgenommen, weil der Wikipedia-Artikel über ihn die Falschinformation verbreitete, er sei ein Terrorist.

April Entfernung des Logos der TU München als sogenannte „Office Action“ - ein Eingriff der Wikimedia Foundation in die Inhalte der Wikipedia, oft wegen juristischer Gründe - aufgrund ungeklärter Rechtslage, nach Klärung derselben am 29. Dezember 2009 wiederhergestellt.

20. April Tagung „Wikipedia in den Wissenschafen“ am Historischen Seminar der Universität Basel.

14. August Veröffentlichung des Software-Werkzeugs „WikiScanner“ durch Virgil Griffith vom California Institute of Technology (Cal Tech), das es erlaubt, IP-Adressen bestimmten Unternehmen und Organisationen zuzuordnen.

24./25. August Die 2. Wikipedia-Academy findet in Mainz statt, dabei wird erstmals ein Wikipedia-Artikel („Ludwig Feuerbach“ von Josef Winiger) mit der Johann-Heinrich-Zedler-Medaille ausgezeichnet. Der Wettbewerb wurde vom Verein Wikimedia Deutschland in $\mathrm{Zu}$ sammenarbeit mit der Spektrum der Wissenschaft Verlagsgesellschaft und der Akademie der Wissenschaften und der Literatur Mainz durchgeführt. Eine Jury aus namhaften Geisteswissenschaftlern vergab den Preis (Prof. Dr. Gernot Wilhelm, Prof. Dr. Michael Stolleis, Prof. Dr. Johannes Fried und Prof. Dr. Kurt Gärtner).

30. September Der von Jimmy Wales verfasste Artikel „Mzoli’s“ (kleiner Freizeitkomplex mit Imbiss, Disco und Restaurant in Kapstadt/Südafrika) wird nach 22 Minuten mit der Begründung fehlender Relevanz gelöscht und löst einen Streit um die Rechte des Gründers aus. 


\section{Phase zunehmender äußerer Beeinflussung (seit 2008)}

\section{8}

10. Januar

26. Februar

April

30. April

6. Mai

20./21. Juni

Jahresmitte

23. Juli
Sperrung des Zugangs zur usbekischen Wikipediaversion bis zum 5. März 2008.

Vereinheitlichung der Zitierung aus den Monumenta Ger-

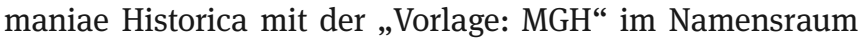
„Vorlage“.

Die für diesen Monat geplante Veröffentlichung des Projektes „Brockhaus Online“ mit 300.000 von der Brockhaus-Redaktion geprüften Texten und mehr als vier Millionen Bildern wird nicht freigeschaltet. ${ }^{8}$ Die deutschsprachige WikipediaVersion zählte damals etwa 730.000 Artikel.

In Syrien wird der Zugang zur arabischsprachigen Wikipedia bis 13. Februar 2009 blockiert, andere Sprachversionen bleiben zugänglich.

Einführung der gesichteten Versionen in der deutschsprachigen Wikipedia zum Schutz vor Vandalismus. Über 13.000 freiwillige Autoren erreichten bisher den Sichterstatus.

Die 3. Wikipedia-Academy findet in Kooperation mit der Berlin-Brandenburgischen Akademie der Wissenschaften in Berlin unter dem Motto „Mathematik, Gesellschaft und Wikipedia“ statt.

Aufgrund konzertierter Aktionen von Einzelnutzern verfügen die Ortsartikel zum polnischen „Kurów“ (ca. 2.870 EW) und zum deutschen „Uetersen“ (ca. 17.200 EW) mit 224 bzw. 228 Interwikilinks über die meisten Sprachversionen für Einzelorte. Später überholte das brasilianische „Curitiba“ (ca. 1,75 Mio. EW) mit 239 Sprachversionen.

Einführung der enzyklopädischen Datenbank „Knol. A unit of Knowledge“ durch Google als Alternative zu Wikipedia. Nach 100.000 Artikeln wurde das Projekt am 30. April 2012 geschlossen und am 1. Oktober 2012 gelöscht.

8 Hoffmann, Björn: Ein Blick zurück von der anderen Seite der Diskette, in: Alles über Wikipedia und die Menschen hinter der größten Enzyklopädie der Welt, hrsg. von Wikimedia Deutschland e.V., Hamburg 2011, S. 31-35. 
31. Juli

15. August

15. September

Oktober

Oktober

13. November

4. Dezember

5. -9 . Dezember
Wiederfreigabe der bis dahin gesperrten chinesischen Wikipedia im Rahmen der Ankunft ausländischer Journalisten zu den Olympischen Spielen in Peking. Die Seiten werden nach Gesprächen zwischen dem Internationalen Olympischen Komitee und den chinesischen Organisatoren der Spiele „entsperrt“.

„MerlBot“, der fleißigste Bot (automatisiertes Skript) der deutschsprachigen Wikipedia, wird aktiviert.

Ein 992 Seiten umfassendes einbändiges Lexikon der 25.000 meistaufgerufenen Wikipedia-Artikel erscheint im Bertelsmann-Lexikon-Institut des Wissen-Media-Verlages unter der GNU-Lizenz für freie Dokumentation. Die Textinhalte basieren auf überarbeiteten Versionen aus dem Artikelraum der deutschsprachigen Wikipedia.

Sperrung des thailändischen Wikipedia-Artikels über Thailands König „Bhumibol Adulyadej“ wegen Majestätsbeleidigung.

Tagung „Neue Formen wissenschaflicher Zusammenarbeit durch kollaborative Medien - Wie Wikipedia und andere Wikiprojekte die (Geistes-)Wissenschafen verändern (können)“ auf Gut Siggen in Ostholstein. ${ }^{9}$

Lutz Heilmann, Abgeordneter des Deutschen Bundestages für die Partei „Die Linke“, lässt den Zugang zur deutschsprachigen Wikipedia über wikipedia.de durch eine einstweilige Verfügung des Landesgerichtes Lübeck sperren, weil in „seinem“ Artikel steht, dass er früher hauptamtlicher Mitarbeiter des Ministeriums für Staatssicherheit der DDR gewesen sei.

Kooperationsvertrag zwischen Wikimedia Deutschland e.V. und dem deutschen Bundesarchiv über die kostenlose Bereitstellung von mehr als 80.000 Bildern durch das Bundesarchiv unter der Lizenz CC BY-SA 3.0 in Wikimedia Commons. Die Zusammenarbeit endet 2010. ${ }^{10}$

In Großbritannien wird der Artikel „Virgin Killer“ über das gleichnamige Album der deutschen Rockband Scorpions und

9 Vgl. http://de.wikipedia.org/wiki/Wikipedia:Siggen (29.5.2011).

10 Sander, Oliver: „Der Bund mit Wiki“ - Erfahrungen aus der Kooperation zwischen dem Bundesarchiv und Wikimedia, in: Der Archivar 63 (2010), H. 2, S. 158-162. 
das damit verbundene Coverfoto „File:Virgin Killer.jpg“ blockiert. Als Grund wird angegeben, das Bild sei „potentially illegal“" und verstoße gegen den Protection of Children Act 1978.

Dezember

Die niederländische Wikimedia-Vertretung gewinnt gegen die einstweilige Verfügung eines Unternehmers, den Artikel zu seiner Person löschen lassen wollte, weil darin eine Verbindung mit dem kriminellen Willem Holleeder genannt wurde.

2009

Jahresverlauf

Bidirektionale Verlinkung von 370.000 Personenartikeln der Wikipedia mit der Personennamendatei (PND). ${ }^{11}$

22. Januar

Der Präsident der Encyclopædia Britannica, Jorge Cauz, gibt bekannt, dass jedermann diese Enzyklopädie im Internet erweitern könne, die Änderungen jedoch von einem Administrator freigegeben werden müssen, bevor sie in der Internetversion erscheinen.

Februar

Als Karl-Theodor Maria Nikolaus Johann Jakob Philipp Franz Joseph Sylvester Freiherr von und zu Guttenberg am 10. Februar 2009 Bundesminister wird, trägt ein unregistrierter Nutzer als elften Vornamen „Wilhelm“ in den Artikel der deutschsprachigen Wikipedia ein. Zahlreiche überregionale Zeitungen und Zeitschriften übernehmen dies ungeprüft. Als verschiedene Wikipedianer den falschen „Wilhelm“ später aus dem Artikel löschen wollen, wird das mit dem Verweis auf diese Zeitungseinträge rückgängig gemacht.

März

Kooperationsvertrag mit der Sächsischen Landes-, Staatsund Universitätsbibliothek Dresden. Diese stellt 250.000 Bilddateien aus ihrer Abteilung „Deutsche Fotothek“ für die freie Nutzung auf Wikimedia Commons zur Verfügung. ${ }^{12}$

10. Mai Einrichtung des Projektes „Marjorie-Wiki“, um (noch) nicht relevante und daher gelöschte Artikel aus der Wikipedia aufzubewahren.

15. Mai „Wolfram Alpha“, ein auf der Software „Mathematica“ basierender Internetdienst zum Auffinden und Darstellen von

11 Gärtner, Kurt: Wikipedia und Wissenschaft aus Sicht der Akademieforschung, in: Alles über Wikipedia, S. 269-272, hier S. 272.

12 Vgl. http://commons.wikimedia.org/wiki/Commons:Deutsche_Fotothek/de (20.2.2015). 
28. Mai

26. Juni

August

11. September

Oktober

9./10. Oktober

19. Oktober

27. Dezember

2010

30. Januar
Informationen, der von Wolfram Research entwickelt wurde, wird online zugänglich.

In der englischsprachigen Wikipedia kommt es zum Schiedsverfahren gegen und Verbot von Artikeländerungen durch die Religionsgemeinschaft Scientology.

Umstellung aller Wikipedia-Projekte von der Lizenz GFDL (GNU Freie Dokumentationslizenz/GNU Free Documentation License) auf eine Doppellizenzierung GFDL und CC BY-SA 3.0. Die Volltexte aus dem Wikipedia-Schwesterprojekt „Wikisource“ werden im Katalog der Universitäts- und Stadtbibliothek Köln nachgewiesen.

Bildspende von über 49.000 Abbildungen durch das Königliche Niederländische Tropeninstitut.

Da die Namensnennung der beiden Mörder des 1990 ermordeten bayerischen Schauspielers Walter Sedlmayr per Gesetz verboten war, forderen Anwälte bei deren Freilassung von der Wikimedia Foundation eine Unterlassungserklärung, um die Namen aus der englischsprachigen Wikipedia zu verbannen. Tagung „Web 2.0 und Geschichtswissenschaft ,Social Networking' als Herausforderung und Paradigma“"

Einrichtung des Online-Glossars „twick.it“, bei dem auch viele Wikipedia-Autoren mitschreiben. Nach 20.000 Einträgen mit jeweils maximal 140 Zeichen zum 1. August 2012 wieder eingestellt.

Die deutschsprachige Wikipedia überschreitet mit „Ernie Wasson“ von Benutzer „JFKCom“ die Marke von 1 Mio. enzyklopädischer Artikeln.

Das Lemma „Chiara Ohoven“ wird bis 30. Januar 2022 vor Neuanlage geschützt, nachdem der Artikel seit 17. August 2005 mindestens sieben Löschdiskussionen samt Löschungen und entsprechende Wiederherstellungen erlebt hat. ${ }^{13}$

13 Nach den Eintragungen im Löschlogbuch müsste dies der Artikel mit den häufigsten Wiedergängern sein: https://de.wikipedia.org/w/index.php?title=Spezial:Logbuch\&page=Chiara Ohoven, 23. 2. 2015. Die letzte Löschdiskussion hatte eigentlich ergeben, den Artikel zu behalten. Vgl. https://de.wikipedia.org/wiki/Wikipedia:L\%C3\%B6schkandidaten/23._November_2006\#Chiara_ Ohoven_.28gel.C3.B6scht.29 (23.2. 2015). 
24. März

Mai

25. Juli

6. September

24. -26. September

31. Oktober

19./20. November

3. Dezember

14. Dezember

2011

14. Januar
Die europäischen Wikipedia-Server fallen wegen Überhitzung kurzzeitig aus und sämtlicher Traffic muss über die Server in Florida laufen.

Die Löschung diverser Bilder mit sexuellen Darstellungen auf Wikimedia Commons durch Jimmy Wales löst einen „PornoStreit“ aus. In den darauffolgenden Diskussionen, einer Petition sowie einem Antrag auf Entzug seiner Gründerrechte gibt Wales freiwillig einige seiner Rechte $a b$.

Werkstattgespräch „Wikipedia und die Geschichtswissenschafen. Zur Praxis und Theorie eines aktuellen Phänomens“ an der Universität Wien.

Bildspende von über 13.000 Abbildungen durch das niederländische Nationaal Archief.

Konferenz „Wikipedia: Ein kritischer Standpunkt“ in der Universitätsbibliothek Leipzig sowie im Geisteswissenschaftlichen Zentrum der Universität Leipzig.

Einrichtung von „Wiki-Watch“ an der Arbeitsstelle im Studien- und Forschungsschwerpunkt „Medienrecht““” der Juristischen Fakultät der Europa-Universität Viadrina Frankfurt/O. unter der Leitung von Wolfgang Stock und Johannes Weberling mit dem Ziel die „Wissens-Ressource Wikipedia transparenter zu machen"14.

Die 3. Wikipedia-Academy 2010 findet an der Goethe-Universität Frankfurt/M. statt.

Einrichtung von „Europäische Geschichte Online“, herausgeben vom Leibniz-Instituts für Europäische Geschichte Mainz und langzeitarchiviert von der Bayerischen Staatsbibliothek in München.

In Kuba wird „EcuRed“ (Enciclopedia cubana en la Red = Kubanische Enzyklopädie im Netz) auf Wikibasis, als „revolutionäre, sozialistische und bolivarische“ Alternative zu Wikipedia gestartet (2014: über 140.000 Artikel).

Einrichtung des Projektes „Wikibu.ch“ an der Pädagogischen Hochschule Bern, das Unterstützung bei der Beurteilung von Wikipedia-Artikeln geben soll. 
16. Januar

2. März

10./11. Juni

Sommersemester

September

Herbst

4. Oktober
Die erste von elf Löschdiskussionen zum Lemma „Sarah Knappik“ findet statt, als Ergebnis bleibt der Artikel über das deutsche Model erhalten. ${ }^{15}$

Strafanzeige mit dem Vorwurf der Urheberrechtsverletzung gegen verschiedene Wikipedia-Nutzer bezüglich der Lemmata „Bancor“ und „Die wirtschaftliche Neuordnung Europas“, nach Anhörung und Stellungnahme wurde das Verfahren im Mai von der Staatsanwaltschaft Hamburg eingestellt.

Veranstaltung „Wikipedia trifft Altertum. Freies Wissen, Neue Medien, populäre Wissensvermittlung und Enzyklopädien in den Altertumswissenschaften“ an der Georg-August-Universität Göttingen.

Das „Wikipedia Ambassador Programm“ wird aufgelegt und in Pilotprojekten an verschiedenen Universitäten in Kanada, den USA ${ }^{16}$, Brasilien und Indien getestet. ${ }^{17}$

Das „Wikipedia Ambassador Programm“ wird in Deutschland als „Wikipedia-Hochschulprogramm“ von Wikimedia Deutschland in Kooperation mit fünf Universitäten/Hochschulen Deutschland (Halle/S., Marburg, München, Potsdam, Stuttgart) gestartet und Ende 2012 ohne Begründung wieder eingestellt.

Der zum 10. Jahrestag entstandene Sammelband „Alles über Wikipedia und die Menschen hinter der größten Enzyklopädie der Welt" mit 100 Beiträgen von Wikipedia-Autoren ${ }^{18}$ wird auf der Frankfurter Buchmesse vorgestellt.

Protest der italienischen Wikipedia für 24 Stunden gegen ein Gesetz der Regierung Berlusconi. ${ }^{19}$

15 Löschanträge wurden gestellt, bearbeitet und abgelehnt am 16., 19., 20., 21., 22., 26., 27. , 28., 30. Januar, 17. Februar und 22. Juli 2011.

16 Vgl. http://en.wikipedia.org/wiki/Wikipedia:United_States_Education_Program/Courses/Past (4.4. 2012).

17 Vgl. http://outreach.wikimedia.org/wiki/Wikipedia_Campus_Ambassador (4.4. 2012).

18 Alles über Wikipedia und die Menschen hinter der größten Enzyklopädie der Welt, hrsg. von Wikimedia Deutschland e.V., Hamburg 2011.

19 Vgl. https://it.wikipedia.org/wiki/Wikipedia:Comunicato_4_ottobre_2011/de (3.3.2015). 


\section{Phase fortschreitender Automatisierung (seit 2012)}

\section{2}

10. Januar

18. Januar

März

13. März

April

April

April

16. Mai

Die PR-Abteilung des Vatikans kopiert die Biographien von 22 durch Papst Benedikt XVI. neu ernannten Kardinälen für deren öffentliche Vorstellung aus der italienischen Wikipedia, ohne diese als Quelle anzugeben.

Protest der englischsprachigen Wikipedia für 24 Stunden gegen zwei Gesetzesvorschläge im US-Congress, den „Stop Online Piracy Act“ (SOPA) und den „PROTECT IP Act“ (PIPA). Einige Abgeordnete ändern ihre Meinung.

Entscheidung des Landgerichtes Berlin zu Gunsten der Erben von Vicco von Bülow (alias Loriot), wonach die Wikipedia vier (am 3. Januar 2011 erschienene) Wohlfahrtsmarken mit Motiven von Loriot („Das Frühstücksei“, „Herren im Bad“, „Auf der Rennbahn“ und „Der sprechende Hund“) nicht zeigen darf. Die Abbildungen der Postwertzeichen waren bereits im Herbst 2011 im Rahmen einer Office Action nach einer einstweiligen Verfügung entfernt worden.

Bekanntgabe der endgültigen Einstellung der gedruckten Encyclopædia Britannica.

Die bisher getrennten Normdateien „Gemeinsame Körperschaftsdatei“ (GKD), „Personennamendatei“ (PND) und „Schlagwortnormdatei“ (SWD) sowie die „EinheitssachtitelDatei des Deutschen Musikarchivs“ (DMA-EST-Datei) werden zur „Gemeinsamen Normdatei“ (GND) zusammengeführt.

Beginn der Umsetzung des Projektes „Wikidata“, das erstmals im September 2004 von Erik Möller vorgeschlagen und anhand eines von Magnus Manske implementierten Prototyps demonstriert worden war. Die Datenbank stellt als gemeinsame Quelle allgemeingültige Datentypen wie Lebensdaten bereit, die in allen Artikeln der Wikimedia-Projekte verwendet werden können.

Der Benutzer Justin Knapp aus Indianapolis, Indiana fügt als erster Nutzer mehr als 1 Mio. Veränderungen (Edits) zur Wikipedia hinzu.

Die Einführung von Googles Knowledge Graphen (der grundlegende Daten anzeigt) in Google.com führt zu einem 
29. Juni-1. Juli

10. Juli

30. Oktober

18. November

\section{3}

27. Januar

6. März

16. März

4. April

20. April

14./15. Juni

Juli
Rückgang des Aufrufs von Wikipedia-Artikeln; am 4. Dezember folgen die Google-Versionen in Spanien, Frankreich, Deutschland, Portugal, Japan, Russland und Italien.

Die 4. Wikipedia-Academy findet in Kooperation mit dem Alexander von Humboldt Institut für Internet und Gesellschaft sowie der Freien Universität Berlin unter dem Motto „Research and Free Knowledge“ in Berlin statt.

24-stündiger Protest der russischsprachigen Wikipedia gegen den Gesetzesentwurf der Duma „Über den Schutz von Kindern vor Informationen, die Gesundheit und Entwicklung schaden“.

Start der frei bearbeitbaren Datenbank „Wikidata“, die als gemeinsame Quelle grundlegende Daten für WikimediaProjekte bereitstellen soll.

Mit „Baracke Wilhelmine“ von Benutzer „Jocian“ überschreitet die deutschsprachige Wikipedia die Marke von 1,5 Mio. enzyklopädischen Artikeln.

Der Asteroid 274301 erhält offiziell den Namen „Wikipedia“. Die in „Wikidata“ zentral gesammelten Sprachlinks stehen in allen Sprachversionen zur Verfügung.

Symposium „Wikidata trifft Archäologie“ in der Bibliothek des Auswärtigen Amtes am Werderschen Markt in Berlin.

Der französische Inlandsgeheimdienst DCRI erzwingt von Rémi Mathis, einem Administrator der französischsprachigen Wikipedia, unter Androhung von Untersuchungshaft die Löschung des französischen Wikipedia-Artikels „Station hertzienne militaire de Pierre-sur-Haute“. Der nach kurzer Zeit wiederhergestellte Artikel wird anschließend in 32 weitere Sprachen übersetzt.

Verdoppelung der Zahl der niederländischen Wikipedia-Artikel innerhalb von 18 Monaten von 768.520 auf 1.548 .591 durch den Einsatz von Bots (automatisierten Skripten).

Die niederländischsprachige Wikipedia überholt mit etwa 1.595.000 Artikeln die deutschsprachige Wikipedia und wird damit zur zweitumfangreichsten Sprachversion nach der englischsprachigen.

Der VisualEditor wird eingeführt, um die Bearbeitung der Artikel zu erleichtern. 


\section{4}

3. März

23. April

Mai

30. Juli

Juli und August

4. August

17. August
Jimmy Wales empfiehlt Studierenden, Akademikern oder anderen Menschen, die auf professioneller Ebene Informationen sammeln und verwerten, Wikipedia nicht zu zitieren. Wikipedia biete Hintergrundwissen und erlaube, sich bei einem Thema $\mathrm{zu}$ orientieren, aber es sei kein Fachmagazin mit Peer-Review. ${ }^{20}$

Wörtliche, aber nicht entsprechend gekennzeichnete Passagen aus der Wikipedia finden sich im 2013 erschienen Werk „Große Seeschlachten: Wendepunkte der Weltgeschichte von Salamis bis Skagerrak“ des Verlages C.H. Beck. Der Verlag zieht das Buch zurück, nachdem eine nachträgliche Überprüfung die Vorwürfe gegen einen der Autoren teilweise bestätigt hat.

Der Europäische Gerichtshof (EuGH) in Luxemburg (Rechtssache C-131/12) verpflichtet die Suchmaschine Google dazu, Verweise auf Webseiten mit sensiblen persönlichen Daten aus seiner Ergebnisliste zu streichen und stärkt damit das „Recht auf Vergessen“ im Internet.

Die niederländische Wikipedia wird bei der Marke von ca. 1,78 Mio. Artikeln von der Anzahl der ebenfalls botgenerierten Artikel der schwedischsprachigen Wikipedia überholt und am 6. Februar 2015 bei 1,81 Mio. Artikeln wieder von der benutzerverfassten deutschsprachigen Version.

Im Rahmen von Meinungsverschiedenheiten um den sogenannten „Medienbetrachter“ kommt es zum Einsatz des sogenannten „Superschutzes“ an der Datei „MediaWiki:Common.js“; wodurch gegen einen deutschsprachigen Community-Beschluss der „MediaViewer“ durchgesetzt wird. Google entfernt erstmals Links zu Wikipedia-Artikeln aus seinem Suchindex. Jimmy Wales, Mitglied des zehnköpfigen Gremiums, welches Google bei der Durchführung der Löschanträge berät, nennt das EuGH-Urteil in einem BBCInterview die weitreichendste Internet-Zensur, die er bis dahin gesehen habe.

Der Vertrieb der Brockhaus-Enzyklopädie wird endgültig eingestellt.

20 Vgl. http://www.heise.de/tr/artikel/Studenten-sollten-uns-nicht-zitieren-2158448.html. 
23.-26. September Auf dem 50. Deutschen Historikertag in Göttingen findet erstmals eine Sektion zu „Wikipedia und Geschichtswissenschaften" statt.

1. Oktober Einführung der Panoramafreiheit in Russland, durch die DUMA. Eine im Jahr 2009 begonnene Initiative der Vereins Wikimedia Russland hatte zur Gesetzesänderung geführt, die auch den Status freier Lizenzen in der russischen Föderation ermöglicht.

22. Oktober Enthüllung des weltweit ersten Denkmals für Wikipedia, entworfen vom armenischen Bildhauer Mihran Hakobyan (Kosten ca. 11.000 Euro) in der westpolnischen Stadt Slubice.

27. Oktober Nach einem verlorenen Markenrechtsstreit gegen Wiki-Watch am 26. Oktober gibt die Wikimedia Foundation alle bisher nicht unter freier Lizenz stehenden eigenen Projektlogos unter CC BY-SA 3.0-Lizenz frei.

November

Bekanntgabe der 2009 eröffneten „Bibliothek des Präsidenten B. N. Jelzin“, eine russischsprachige Onlineenzyklopädie als Alternative zu Wikipedia gründen zu wollen, da Wikipedia „nicht in der Lage [sei], detaillierte und zuverlässige Informationen über die Regionen Russlands und das Leben im Land zu geben. “21

16. Dezember Freebase, eine Online-Sammlung strukturierter Daten, die 2007 von der Firma Metaweb eingeführt und am 16. Juli 2010 von Google gekauft worden war und die eine der Grundlagen des Google-Knowledge-Graphen ist, soll bis Mitte 2015 geschlossen und die Daten an das Projekt Wikidata übergeben werden.

2015

15. Januar

Bekanntgabe der niederländischen Praemium Erasmianum Stiftung, den seit 1958 verliehenen Erasmuspreis mit einem Preisgeld von 150.000 Euro an die Wikipedia-Community zu verleihen.

10. März Jimmy Wales und Lila Tretikov kündigen in „The New York Times“ an, die National Security Agency (NSA), den größten Auslandsgeheimdienst der USA, wegen gesetzlich nicht gedeckter Massenüberwachung von Wikipedianutzern/innen zu verklagen. Wikimedia bereitet die Klage gemeinsam mit

21 Vgl. http://www.prlib.ru/en-us/events/Pages/Item.aspx?itemid=1077 (3.3.2015). 
Bürgerrechtsgruppen (ACLU), Amnesty International und Human Rights Watch vor.

9. Juli Das EU-Parlament lehnte einen Vorschlag (vom 25. Juni) zur Panoramafreiheit ab, der das Fotografieren öffentlicher Gebäude und Kunstwerke in allen EU-Staaten mit einem generellen Erlaubnisvorbehalt einschränken wollte. Eine Petition zur Erhaltung der Panoramafreiheit hatte über 500.000 Mitzeichner erreicht. 
